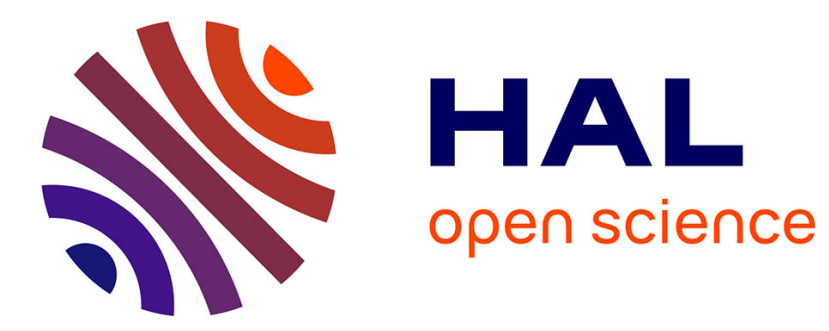

\title{
Modeling of induced current into the human body by low-frequency magnetic field from experimental data
}

Riccardo Scorretti, Noël Burais, Laurent Nicolas, Alain Nicolas

\section{To cite this version:}

Riccardo Scorretti, Noël Burais, Laurent Nicolas, Alain Nicolas. Modeling of induced current into the human body by low-frequency magnetic field from experimental data. IEEE Transactions on Magnetics, 2005, 41 (5), pp.1992-1995. hal-00140334

\section{HAL Id: hal-00140334 \\ https://hal.science/hal-00140334}

Submitted on 6 Apr 2007

HAL is a multi-disciplinary open access archive for the deposit and dissemination of scientific research documents, whether they are published or not. The documents may come from teaching and research institutions in France or abroad, or from public or private research centers.
L'archive ouverte pluridisciplinaire HAL, est destinée au dépôt et à la diffusion de documents scientifiques de niveau recherche, publiés ou non, émanant des établissements d'enseignement et de recherche français ou étrangers, des laboratoires publics ou privés. 


\title{
Modeling of Induced Current Into the Human Body by Low-Frequency Magnetic Field From Experimental Data
}

\author{
Riccardo Scorretti, Noël Burais, Laurent Nicolas, and Alain Nicolas \\ CEGELY_UMR CNRS 5005, Ecole Centrale de Lyon, 69134 Ecully cedex, France
}

\begin{abstract}
The induced currents into the human body by a low-frequency magnetic field are computed using three-dimensional finite elements and a special $\phi$-A formulation. This magnetic field is generated by a real (possibly unknown) power system. The stray field is characterized by an equivalent multipole, fitted from some local measurements of the field, which is a good compromise between accuracy and number of degrees of freedom. The methodology is validated on a test-device using the software FLUX3D
\end{abstract}

Index Terms-Equivalent source, finite-element method, human exposure, inverse problem, low-frequency magnetic stray fields.

\section{INTRODUCTION}

$\mathbf{T}$ HE increasing usage of the electric energy in the daily life raises the question of the effects of the exposure to the electromagnetic fields (EMF) on the human health. From a physical point of view, the main effect of the exposure to low-frequency (up to $1 \mathrm{MHz}$ ) electromagnetic fields is the generation of induced currents inside the human body [1]. While the short-term effects of this exposure are well known, there is no convincing evidence of (possibly hazardous) long-term effects of the electromagnetic fields on the human health [2]. For this reason, a number of industrial standards for limiting the EMF exposure have been proposed [3]-[5]. In the case of the low-frequency (LF) magnetic fields, these limitations have been defined in terms of basic restrictions for the induced current density, and reference levels for the EMF, ${ }^{1}$ as a function of the frequency.

Thus, the quantification of the induced currents by EMF is a major issue, both for the medical research and for the definition of industrial standards. Unfortunately, these induced currents are extremely hard to measure in vivo, so that they have to be computed numerically. Several works exist in the scientific literature on the computation of induced currents into the human body using different methods like finite element [6], [7], finite-difference time-domain [8], or the impedance method [9]. In most of these works, great attention is paid to the model of the human body, whereas the source of the magnetic field is generally quite simple: the field is often supposed to be uniform, or at most generated by a wire system. In a previous work [10] we presented a special quasi-static $\phi$-A formulation, which allows to compute separately the magnetic field generated by a power system, and the induced currents into the human body. Hence, the exposure to realistic power systems can be modeled,

Digital Object Identifier 10.1109/TMAG.2005.846276

${ }^{1}$ The basic restrictions are the maximum levels of the induced current, which should not be exceeded in any case. The respect of the reference levels for the EMF is not mandatory, but it guarantees that, even in the worst case, the basic restrictions are not exceeded. provided that an accurate model of the radiating system is available. Unfortunately, in some cases of practical interest, the details of the power system are not well known. In these cases, one wonders how to substitute the radiating power system with an equivalent source, which could be obtained from some measurements of the stray field. The purpose of this paper is to present a methodology to compute the induced currents into the human body by a completely unknown power system.

\section{Methodology}

The methodology consists of three steps. First, the magnetic field generated by the power system has to be measured in some points in the air around the system. Second, an equivalent source is fitted from these measurements. Third, the field of this equivalent source is used to compute the induced current into the human body through a special $\phi$-A formulation.

\section{A. Measurement System}

Assume that the power system generates a periodic field-say a pure $50-\mathrm{Hz}$ signal, for sake of simplicity. Hence, in each point the magnetic field can be represented with a three-dimensional (3-D) complex vector, describing the amplitude and the phase of the field. It is important to stress that both the amplitude and the phase have to be measured [11]. To this aim, we used a couple of one-axis induction coils as sensors. One of these coils is fixed in an arbitrary point on the surface of the power system, and it is used as phase reference. The other one is placed successively in each measurement point; thus, the amplitude and the phase of one component of the magnetic field are obtained. Hence, a 3-D measurement of the field in each point is easily obtained. In order to automate the measurement process, we used a MICROCONTROLE IT6DCA robot system to move the measurement coil (Fig. 1).

\section{B. Estimation of an Equivalent Source}

There exists several models to characterize the magnetic stray field generated by a power system: single or double layer potential [12], multipoles [11], [13], or even a simple dipole [14]. Among these models, we have chosen a third-order spherical 


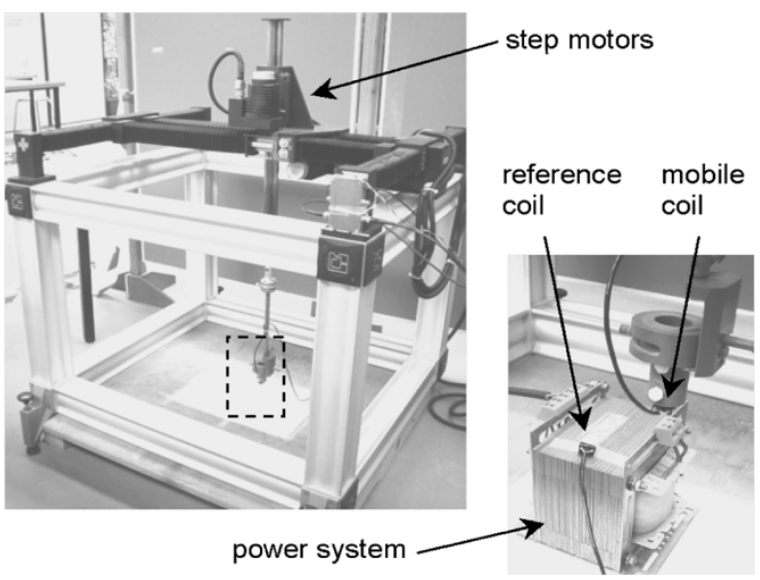

Fig. 1. Measurement system. The mobile coil is placed at the bottom of a bakelite arm, which is moved by three step-motors. The reference coil is fixed onto the power system (example: a single-phase transformer).

multipole as equivalent source, because it has a small number of degrees of freedom (30), and it is yet accurate enough in the air surrounding the power system.

The expression of the magnetic vector potential $\mathbf{A}$ of a multipole located at the origin is

$$
\mathbf{A}=-\mu_{0} \sum_{n=1}^{N} \frac{1}{n}\left(\frac{R}{r}\right)^{n+1} \sum_{m=-n}^{N} C_{n}^{m} \mathbf{r} \times \vec{\nabla} Y_{n}^{m}(\vartheta, \varphi)
$$

where $N$ is the order of the multipole, $Y_{n}^{m}(\vartheta, \varphi)$ are the spherical harmonics, and $C_{n}^{m}$ are complex coefficients which have to be fitted. This equation holds in the free space for any $r \geq R$, where the constant $R$ is chosen in such a way that the power system is inside the sphere $r<R$. One observes that this potential-and thus the magnetic field-depends linearly upon the coefficients. Thus, in principle, the problem of identifying these coefficients can be solved using the standard least-squares method.

Unfortunately the problem is not as simple as it may appear at a first glance. Indeed, it has been shown that in order to obtain a good agreement with experimental data, the position $\mathbf{x}_{0}$ of the multipole is crucial [15]. One observes that the magnetic field depend in a nonlinear way upon $\mathbf{x}_{0}$ through the position vector $\mathbf{r}=\mathbf{x}-\mathbf{x}_{0}$. This problem can be overcome by choosing the position $\mathbf{x}_{0}$ which minimizes the scatter between the field of the multipole and the measurements.

Moreover, when the measurement points cannot be taken all around the power system, the problem can be ill-posed. This can be explained by the argument that when only a fraction of the solid angle is "explored," it is difficult to obtain enough information to characterize the stray field in the whole space. In particular, the worse case is when the measurement points are aligned onto a straight line-which is precisely the most simple way to operate [14]. This problem can be partially overcome by using regularization techniques, like the singular values decomposition [11], or Tikhonov's method [16].

\section{Computation of Induced Currents Into The Human Body}

Once the equivalent multipole has been obtained, the vector potential $\mathbf{A}$ and the flux density $\mathbf{B}$ can be computed through
TABLE I

Conductivities of USUAL HuMAN TISSUES (S/m)

\begin{tabular}{llllll}
\hline \hline & $10 \mathrm{~Hz}$ & $1 \mathrm{kHz}$ & $10 \mathrm{kHz}$ & $100 \mathrm{kHz}$ & $1 \mathrm{MHz}$ \\
\hline Fat & 0.012207 & 0.022404 & 0.02383 & 0.024414 & 0.025079 \\
Muscle & 0.20197 & 0.32115 & 0.34083 & 0.36185 & 0.50268 \\
Bone & 0.075563 & 0.08153 & 0.02043 & 0.083892 & 0.024353 \\
Kidney & 0.05441 & 0.11274 & 0.13774 & 0.17134 & 0.27823 \\
Liver & 0.027714 & 0.04138 & 0.053495 & 0.084568 & 0.18665 \\
Blood & 0.7 & 0.7 & 0.70004 & 0.70292 & 0.82211 \\
Intestine & 0.51113 & 0.53236 & 0.55967 & 0.5942 & 0.86488 \\
Bladder & 0.20278 & 0.20758 & 0.21303 & 0.2189 & 0.23608 \\
\hline \hline
\end{tabular}

(1) in any point of the free space surrounding the power system. Owing to the weak conductivity of the living tissues [17]-[19] (Table I), the variation of the magnetic field due to eddy currents inside the human body (i.e., the "armour reaction") is negligible [6]-[9]. Even for a rather "high" value of the conductivity $\sigma=$ $1 \mathrm{~S} / \mathrm{m}$, the limit of this approximation is found at the frequency $f=1 \mathrm{MHz}[10]$.

Hence, the vector potential $\mathbf{A}$ is assumed to be known. In the harmonic regime one may write

$$
\vec{\nabla} \times \mathbf{E}=-j \omega \vec{\nabla} \times \mathbf{A} \quad \Rightarrow \quad \mathbf{E}=-j \omega \mathbf{A}-\vec{\nabla} \phi
$$

thus the conduction current density may be written as

$$
\mathbf{J}=\sigma \mathbf{E}=-\sigma(j \omega \mathbf{A}+\vec{\nabla} \phi)
$$

where $\phi$ is an unknown electric scalar potential, which has to be computed. The displacement currents are not taken into account. By substituting (3) into the charge conservation law $\vec{\nabla} \cdot \mathbf{J}=0$, one obtains

$$
\vec{\nabla} \cdot(\sigma \vec{\nabla} \phi)=-j \omega \vec{\nabla} \cdot(\sigma \mathbf{A})
$$

This equation holds in any point inside the human body, provided that the conductivity $\sigma$ is continuous. At the interface between different tissues the continuity of the normal component of $\mathbf{J}$ has to be ensured, that is

$$
\left(\mathbf{J}_{2}-\mathbf{J}_{1}\right) \cdot \mathbf{n}=0
$$

and notably at the boundary of the human body one has

$$
\mathbf{J} \cdot \mathbf{n}=0 \Rightarrow \frac{\partial \phi}{\partial \mathbf{n}}=-j \omega \mathbf{A} \cdot \mathbf{n} .
$$

Equations (4)-(6) represent the strong formulation of the problem. It is important to remark that the resolution domain of this problem is bounded to the sole human body-that is, the "outer world" is implicitly taken into account through the vector potential $\mathbf{A}$. This is a major advantage with respect to a classical magnetodynamic formulation (smaller numerical systems, simpler boundary conditions).

This formulation has been implemented using the finite-element method with classical nodal elements [10]. An anatomical model of the human body has been obtained from 59 CT scans. It is made of 33181 nodes and 182650 first-order tetrahedral 

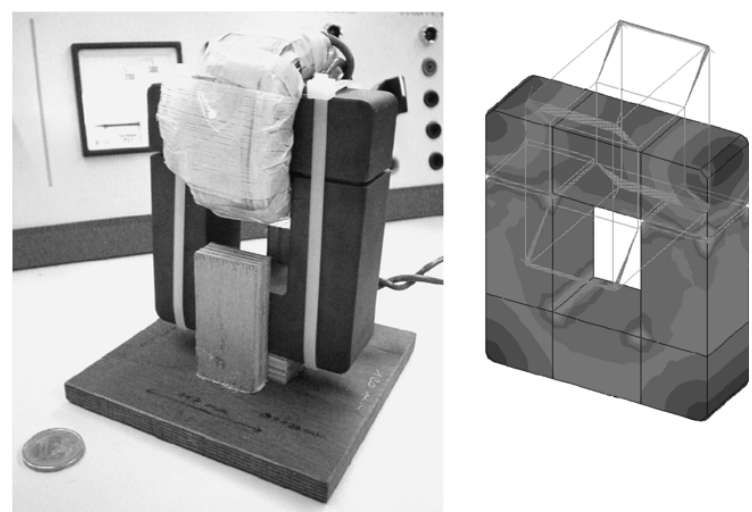

Fig. 2. Test device (left), and the flux density in the core computed using the software FLUX3D (right).

elements, and it is composed of 11 different tissues, characterized by their own conductivities. The computational time is of about 10 min using a 2-GHz ATHLON processor and a memory of $256 \mathrm{MB}$.

\section{VALIDATION}

The methodology has been validated using a simple test device, composed of a coil, a magnetic core, and two air gaps (Fig. 2, left). Actually, a detailed model is available for this test device (Fig. 2, right), so that we have been able to compare the measurements with the numerical results obtained using both the proposed methodology, and the finite-element software FLUX3D. ${ }^{2}$ When using FLUX3D, the whole system (power system + "human body" + air) has to be taken into account at the very same time.

\section{A. Flux Density}

Two sets of measurements have been taken [Fig. 4(a)]:

1) 64 points located on a plane above the test device;

2) 15 points located onto a straight line.

The set \#1 has been used to fit the equivalent multipole, while the set \#2 is used to validate the model. In Fig. 3 the measured flux density (set \#2), the flux density computed using FLUX3D, and the fits obtained from these values using a third-order multipole are plotted. A good agreement is found: the maximum scatter between all these data is at most $10 \%$. Besides, one observes [Fig. 4(b)] that the multipole has been located approximately at the center of the coil, which is a pretty interesting result.

\section{B. Induced Currents}

The current density which is induced into an homogeneous cylinder $(\sigma=0.4 \mathrm{~S} / \mathrm{m})$ has been then computed using both FLUX3D [Fig. 4(a)], and the special $\phi$-A formulation with the field of the equivalent multipole [Fig. 4(b)]. The scatter between the maximum values of $\|\mathbf{J}\|$ computed with the two methods has been found to be less than $5 \%$. Interestingly, one observes that these maximum values of $\mathbf{J}$ are not located where the flux density $\mathbf{B}$ is the more intense.

22D/3D FEM software products developed by INPG/LEG and CEDRAT.

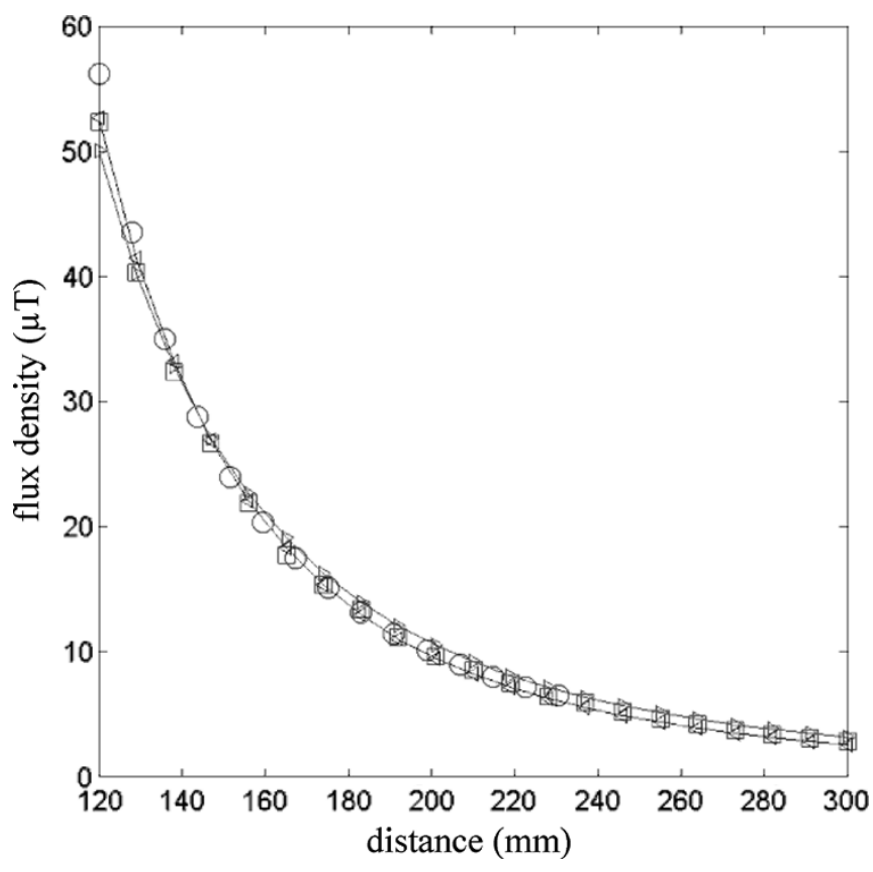

Fig. 3. Flux density along the straight line (set \#2): measurements $(\bigcirc)$, FLUX3D ( $\square$ ), fits using a third-order multipole (continuous lines).

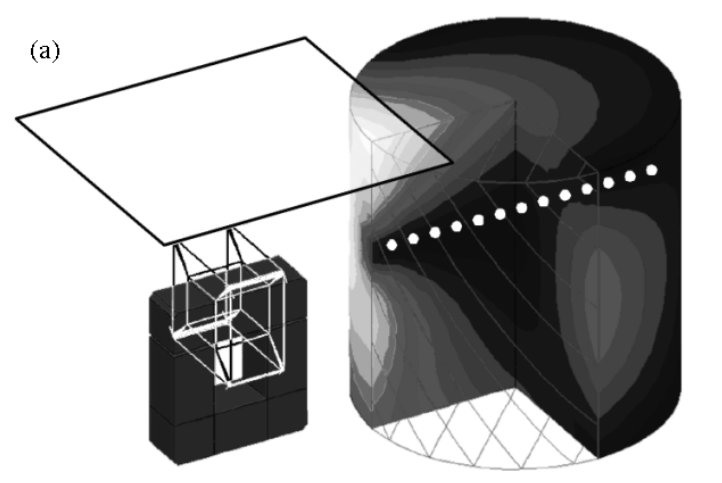

(b)

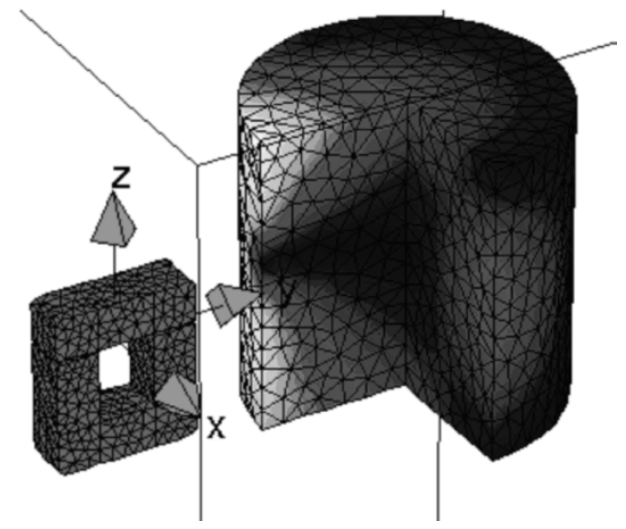

Fig. 4. a) Induced current density into an homogeneous cylinder computed using FLUX3D. The opaque plane and the dotted line represent the measurement points of the set \#1 and \#2 respectively. b) The same current density computed using the proposed methodology. The origin of the axis represents the optimal position $\mathbf{x}_{0}$ of the equivalent multipole.

Finally, the induced currents have been computed using the anatomical model of the human body (Fig. 5). In this case, the same computation using FLUX3D has been impossible owing to the complexity of the model; thus, no direct comparison is available. Nevertheless, one observes some similarities between the 


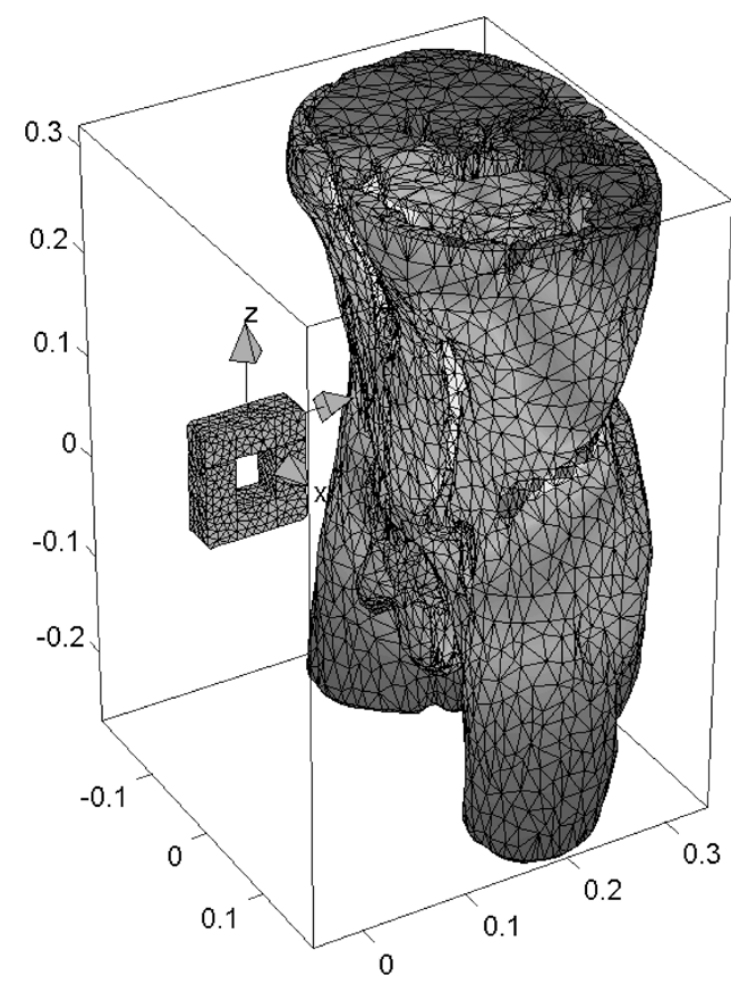

Fig. 5. Induced current density into an anatomical model of the human body.

orders of magnitude and the distribution of the currents computed into the homogeneous cylinder and the anatomical model.

\section{CONCLUSION}

A methodology to quantify the induced current into the human body by an unknown power appliance is presented. The originality of this work consists in the "global" approach (measurement, characterization of the magnetic stray field, computation of the induced currents) to this problem.

However, a great amount of work still has to be done: the feasibility of such a methodology has been proved, but several points have to be improved. First, no quantitative information about the reliability of the equivalent source is available: one wonders how to obtain the standard deviation of the estimation of the fitted coefficients. Some other approaches to the problem of the characterization of the stray fields ought to be investigated. Second, in the computation of the induced currents inside the human body, displacement currents are neglected. Moreover, only the magnetic field is taken into account: for some applications (i.e., high-voltage lines) it would be suitable to take into account both the electric and magnetic fields. However, in the authors' opinion, the extreme challenge with the modeling of induced phenomena into the human body is the experimental validation. This problem is particularly difficult at low frequencies, because of the low energy levels, which make any magnetic or thermal effect extremely hard to be measured.
The proposed methodology could find some major industrial applications (assessment of the respect of the basic restriction, definition of more appropriate industrial standards, etc.).

\section{REFERENCES}

[1] F. S. Barnes, "The effects of time varying magnetic fields on biological materials," IEEE Trans. Magn., vol. 26, no. 5, pp. 2092-2097, Sep. 1990.

[2] M. A. Stuchly, "Electromagnetic fields and health," IEEE Potentials, vol. 12, no. 2, pp. 34-39, Apr. 1993

[3] "Guidelines for limiting exposure to time-varying electric, magnetic and electromagnetic fields (up to $300 \mathrm{GHz}$ )," Health Phys., vol. 74, no. 4, pp. 494-522, 1998.

[4] IEEE Standard for Safety Levels With Respect to Human Exposure to Electromagnetic Fields, 0-3 kHz, IEEE Standard C95.6, 2002.

[5] IEEE Standard for Safety Levels With Respect to Human Exposure to Radio Frequency Electromagnetic Fields, $3 \mathrm{kHz}$ to $300 \mathrm{GHz}$, IEEE Standard C95.1, 1999.

[6] G. A. Mouchawar, J. A. Nyenhuis, J. D. Bourland, L. A. Geddes, D. J. Schaefer, and M. E. Riehl, "Magnetic stimulation of excitable tissue: calculation of induced eddy-currents with a three-dimensional finite-element model," IEEE Trans. Magn., vol. 29, no. 6, pp. 3355-3357, Nov. 1993.

[7] W. Wang and S. R. Eisenberg, "A three-dimensional finite element method for computing magnetically induced currents in tissues," IEEE Trans. Magn., vol. 30, no. 6, pp. 5015-5023, Nov. 1994.

[8] T. W. Dawson, K. Caputa, and M. A. Stuchly, "Organ dosimetry for human exposure to nonuniform $60-\mathrm{Hz}$ magnetic fields," IEEE Trans. Power Del., vol. 14, no. 4, pp. 1234-1239, Oct. 1999.

[9] N. Hayashi, K. Isaka, H. Tarao, and Y. Yokoi, "Numerical calculation of induced electric field and currents on simple models of multimedium biological systems using the impedance method," in 9th Int. Symp. High Voltage Engineering, Graz, Austria, 1995.

[10] R. Scorretti, N. Burais, O. Fabregue, A. Nicolas, and L. Nicolas, "Computation of the induced current density into the human body due to relative LF magnetic field generated by realistic devices," IEEE Trans. Magn., vol. 40, no. 2, pp. 643-646, Mar. 2004.

[11] C. E. Lyon, "Modeling of magnetic fields generated by low frequency sources using multipole techniques," Ph.D. thesis, Washington State University, Pullman, 1994.

[12] M. Legris, "Identification de l'état magnétique d'un système ferromagnétique à partir de mesures du champ proche," Ph.D. thesis, INPG, Grenoble, France, 1996.

[13] J. Lorange, "Couplage des inductances par rayonnement magnétique. Etude théorique et expérimentale," Ph.D. thesis, INPG, Grenoble, France, 2001.

[14] L. E. Zaffanella, T. P. Sullivan, and I. Visintainer, "Magnetic field characterization of electrical appliances as point sources through in situ measurements," IEEE Trans. Power Del., vol. 12, no. 1, pp. 443-450, Jan. 1997.

[15] R. Scorretti, R. Takahashi, L. Nicolas, and N. Burais, "Optimal characterization of LF magnetic field using multipoles," COMPEL, vol. 23, no. 4, pp. 1053-1061, Nov. 2004.

[16] R. Scorretti, D. Coiffard, and N. Burais, "Estimation of an equivalent multipole using Tikhonov regularization," in 7th Workshop OIPE, Grenoble, France, Sep. 2004.

[17] C. Gabriel, S. Gabriel, and E. Corthout, "The dielectric properties of biological tissues: I. Literature,” Phys. Med. Biol., vol. 41, pp. 2231-2249, 1996.

[18] S. Gabriel, R. W. Lau, and C. Gabriel, "The dielectric properties of biological tissues: II. Measurements in the frequency range $10 \mathrm{~Hz}$ to 20 GHz," Phys. Med. Biol., vol. 41, pp. 2251-2269, 1996.

[19] —, "The dielectric properties of biological tissues: III. Parametric models for the dielectric spectrum of tissues," Phys. Med. Biol., vol. 41, pp. 2271-2293, 1996.

Manuscript received June 8, 2004. 